\section{Patients with private health insurance using NHS facilities in preference to private care}

\author{
A C Fairbank, S R Stapleton, P E M Jarrett
}

Department of Surgery,

Kingston Hospital,

Kingston upon Thames, Surrey KT2 7QB

A C Fairbank, MB, senior house officer

S R Stapleton, FRCS, senior house officer

P E M Jarrett, FRCS, consultant

Correspondence to:

Dr A C Fairbank,

Department of

Cardiothoracic Surgery,

John Radcliffe Hospital,

Headington, Oxford OX3 9DU.

BrMed J 1990;300:719
During 1988-9 we noticed that some patients were receiving NHS treatment at this hospital despite being covered by a private health insurance scheme. We therefore studied why such people had chosen NHS care rather than the private alternative.

\section{Patients, methods, and results}

For one week in June 1989 we asked all patients attending the surgical wards, the accident and emergency department (serving a population of about 200000 ), the surgical day unit (which carries out minor operations under local and general anaesthetics), and the surgical outpatient department in the department of surgery at this hospital to complete a questionnaire anonymously. The questionnaire asked whether the patients had a private health insurance policy and, if so, whether they were using their insurance for the attendance. Those not doing so were asked their reasons why. Patients were told that the study was confidential, and they were not put under any pressure to transfer to the private sector.

The questionnaire was completed by 587 patients. Five patients were unable to complete the form and one refused. Between $6 \%$ and $27 \%$ of patients in the four clinical areas held private insurance policies but were not using them. Overall, this represented about $10 \%$ of patients attending the department during the week studied (table).

When asked why they were using the NHS despite being insured for private treatment $42(72 \%)$ patients stated that they were happy with NHS care 25 added that the short waiting list at the hospital had influenced their decision); three had forgotten about their insurance; five were unaware that private facilities existed in Kingston; three were unsure how to use their private policy; and the remaining six patients gave no reason.

Patients with private insurance who attended outpatient clinics or the accident and emergency department did not transfer to the private sector after their consultation or after completing the questionnaire.

Number of patients with private health insurance attending departmen of surgery during one week who were treated privately or by NHS

\begin{tabular}{lccc}
\hline & \multicolumn{3}{c}{ Patients treated Patients treated } \\
& No of patients & privately & by NHS (\%) \\
\hline Surgical ward & 104 & 3 & $6(6)$ \\
Outpatient clinic & 233 & 2 & $19(8)$ \\
$\begin{array}{l}\text { Accident and emergency } \\
\text { department }\end{array}$ & 186 & 5 & $17(9)$ \\
Surgical day unit & 64 & 7 & $17(27)$ \\
\hline Total & 587 & 17 & $59(10)$ \\
\hline
\end{tabular}

\section{Comment}

Kingston Hospital is in an area with several private hospitals that could have catered for the $10 \%$ of patients attending the department of surgery who had private health insurance. Our data suggest that most of these patients chose to remain in the NHS system because they were happy with NHS care, being influenced in particular by a short waiting list; the typical waiting time for admission to wards is one month, for admissions to the surgical day unit two or three weeks, and for non-urgent outpatient appointments two weeks. Presumably if patients with private insurance policies were treated privately waiting lists in the hospital would be shorter still.

Some of the patients with private insurance may not have been paying for it themselves - for example, those belonging to a company scheme; we did not ask about this. Some of these patients might not want to take up the private option. Our results, however, suggest that satisfaction with the NHS rather than a reluctance to use the private sector was the main reason for patients with private health insurance receiving NHS treatment.

We believe that this study has important implications for planning health care facilities.

(Accepted 13 December 1989 )

\section{Comparison of oral preparations of heroin and methadone to stabilise opiate misusers as inpatients}

\section{A H Ghodse, F J Creighton, A V Bhat}

\section{Division of Pyschiatry of} Addictive Behaviour, St George's Hospital Medical School, London SW17 0RE A H Ghodse, FRCPSYCH, chairman of division F J Creighton, MRCPSYCH, research fellow

A V Bhat, Msc, medical statistician

\section{Correspondence to:}

Professor Ghodse.

BrMed f 1990;300:719-20
Many opiate misusers express a preference for heroin over methadone, and possibly more misusers would seek treatment if heroin was given for maintenance and withdrawal rather than methadone mixture. Because of the risks of intravenous administration it is preferable to give all drugs orally. We report what we believe to be the first comparison of oral heroin and oral methadone for stabilising opiate misusers as inpatients.

\section{Patients, methods, and results}

In a double blind study 57 patients who were dependent on opiates and aged $19-42$ (mean $29 \cdot 2$ years) were randomly allocated to receive heroin or methadone mixture $(1 \mathrm{mg} / \mathrm{ml})$ in otherwise identical solutions. A $10 \mathrm{ml}$ aliquot was given whenever signs of physical withdrawal were observed, and the total given to a patient during the first 24 hours was taken as that patient's daily requirement. This was subsequently given in three doses, with further aliquots being given if signs of opiate withdrawal were observed. Subjects who had been comfortable for two successive days were said to be stabilised.

The severity of withdrawal was assessed on the first day by administering a questionnaire before each dose of opiate; the nursing staff's rating of the presence or absence of classic signs of opiate withdrawal; and recordings of physiological variables. Subjective and objective withdrawal scores were calculated for each subject. This procedure was repeated on each subsequent day at $4 \mathrm{pm}$. Subjects were also asked to note if and when they first noticed craving after the dose of opiate given at $8 \mathrm{am}$, and at the end of the stabilisation period they completed another questionnaire aimed at detecting whether they had noticed particular effects due to their treatment.

The table shows the personal characteristics and drug histories of the 57 patients. Thirty six patients completed the stabilisation procedure, 14 of whom received heroin. The mean dose of heroin required for stabilisation $(55 \mathrm{mg})$ was significantly higher than the mean dose of methadone $(36 \mathrm{mg})(t=2 \cdot 78, \mathrm{p}<0 \cdot 01)$, although the mean reported daily doses of heroin in the two groups before admission were not significantly 


\begin{tabular}{lcc}
\hline & $\begin{array}{c}\text { Patients prescribed } \\
\text { heroin } \\
(\mathbf{n}=28)\end{array}$ & $\begin{array}{c}\text { Patients prescribed } \\
\text { methadone } \\
(\mathbf{n}=29)\end{array}$ \\
\hline Mean age (years) & $28 \cdot 4$ & $30 \cdot 4$ \\
No of men:women & $15: 13$ & $23: 6$ \\
Psychological test scores on admission: & $17 \cdot 3$ & $18 \cdot 0$ \\
$\quad$ Beck depression inventory' & $24 \cdot 2$ & $25 \cdot 6$ \\
$\quad$ Taylor anxiety scale & $21 \cdot 7$ & $20 \cdot 2$ \\
Mean age at first use of heroin (years) & $6 \cdot 4$ & $8 \cdot 1$ \\
Mean duration of use of heroin (years) & 19 & 14 \\
No who regularly injected opiates & $1 \cdot 6(0-6)$ & $1 \cdot 8(0-7)$ \\
Mean No of previous treatments (range) & $18(625 \mathrm{mg})$ & $18(721 \mathrm{mg})$ \\
No of patients who reported having used (and mean daily dose): & $4(47 \mathrm{mg})$ & 7 \\
Heroin only & 5 & $\cdot 2$ \\
Methadone only & 1 & 2 \\
Heroin and methadone & & \\
Other opiates & & \\
\hline
\end{tabular}

different $(536 \mathrm{mg}$ and $653 \mathrm{mg}$ ). In both groups there was a strong correlation in patients who had injected heroin between the stated daily dose of illicit heroin before admission and the final dose of opiate required as an inpatient. This relation was much less consistent among those who had smoked heroin.

When objective and subjective withdrawal scores were averaged for each group the only significant difference occurred on the second day, when the mean objective score for the group given heroin was greater $(t=2 \cdot 23 ; \mathrm{p}<0.05)$. There was no significant difference between the two groups in the time of onset of craving, and neither group identified the treatment drug as being like any particular opiate (heroin, methadone, morphine, codeine, Diconal).

\section{Comment}

We found that opiate misusers can be successfully stabilised with either oral heroin or oral methadone and that patients were unable to identify which drug they received. The mean doses required for stabilisation by the two groups indicate that methadone has approximately 1.5 times the potency of heroin when used to prevent withdrawal. Although our study is the first reported direct comparison of oral administration of the two drugs, the result is consistent with what might have been expected from available information.

The patients who received heroin required more aliquots of opiate during the first 24 hours and had significantly higher withdrawal scores on the second day of stabilisation when the drug was given regularly rather than on demand. This may be because heroin has a shorter half life than methadone and more doses are required before blood concentrations are high enough to suppress withdrawal throughout the day.

The consistent relation between the stabilisation dose and the reported daily dose of injected heroin suggests that patients had reported their use fairly accurately and that street heroin currently has a purity of less than $10 \%$. The less consistent relation for patients who had smoked heroin suggests that absorption by this route is erratic.

We thank the patients, the pharmacists in Tooting Bec and Springfield Hospitals, and the staff of the regional drug dependence treatment and research unit, Springfield Hospital The work was supported by a research grant from Bayer UK

1 Martin WR, Jasinski DR, Haertzen CA, et al. Methadone-a re-evaluation. Arch Gen Psychiatry 1978;28:286-95.

2 Gilman AG, Goodman LS, Rail TW, Murad F, eds, Goodman and Gilman's the pharmacological basis of therapeutics. 7th ed. New York: Macmillan, 1985: $491-531$.

3 Beck AT, Ward AH, Mendelson M, Mock J, Erbaugh J. An inventory for measuring depression. Arch Gen Psychiatry 1961;4:561-71.

+ Taylor JA. A personality scale of manifest anxiets. Foumal of A thormal and Social Psychology 1953:48:285-90.

(Accepled 15 December 1989

\section{Deaths from asthma in the mentally handicapped}

\author{
James M Stuart, Sarah L Stewart-Brown, \\ John Harvey, Kieran Morgan
}

\section{Southmead Health}

Authority, Southmead

Hospital, Bristol BS16 5NB

James M Stuart, MFCM, senior registrar in public health medicine

Sarah L Stewart-Brown, MFCM, consultant in public health medicine

John Harvey, MRCP, consultant physician

Kieran Morgan, MFCM,

director of public health

Correspondence to: $\mathrm{Dr}$ James M Stuart, Gloucester

Health Authority

Gloucester GLl 1LY.

BrMed Y 1990;300:720-1
A review of avoidable causes of death in the Southmead health district identified a high mortality from asthma among 5-44 year olds. ${ }^{1}$ Mortality from asthma in the district was twice the national figure, with an average of two deaths a year. Research has identified potentially avoidable factors that contribute to death from asthma - namely, failure by the patient, relative, or attending medical staff to appreciate the severity of the attack; failure to reach hospital sufficiently quickly; and failure of medical staff to give adequate treatment. ${ }^{2} \mathrm{We}$ conducted a confidential inquiry to examine whether such contributory factors could have been partly reponsible for the deaths of residents in Southmead Health Authority.

\section{Subjects, methods, and results}

We obtained copies of the death certificates of all residents in Southmead Health Authority who died from asthma aged $<45$ between 1974 and 1986 from the Office of Population Censuses and Surveys; we also examined these subjects' medical records.

The age and sex distributions of the subjects, place of death, and identified failures of management (for example, underprescription of steroids) did not explain the high mortality from asthma. Four of the 24 deaths, however, occurred in residents of hospitals for the mentally handicapped, and in four other cases learning difficulties had been documented. Thus a third of those who died had mental disability - a group estimated to comprise $0.6 \%$ of the district's population. Six of these eight subjects died from acute asthmatic attacks and two from complications of chronic asthma (table). A reduction in the dose of oral steroids in case 2 may have contributed to the patient's death. In case 4 asthma had not been recognised until the final illness, which was treated with only low doses of oral salbutamol. In case 7 a recommendation by staff at a chest clinic to prescribe long term oral steroids had not been implemented.

\section{Comment}

The mentally handicapped have not previously been recognised to be at a high risk of dying from asthma. We found no correlation between districts with a high mortality from asthma and districts, such as Southmead, with a high number of beds for the mentally handicapped per 1000 population..$^{13} \mathrm{~A}$ study in the West Midlands region, however, showed an association between long stay mental hospitals (those for the mentally ill and those for the mentally handicapped) and all avoidable causes of death, particularly those classified as pneumonia and bronchitis. ${ }^{+}$Our findings may therefore illustrate a poorly recognised national phenomenon.

Difficulty and delay in diagnosis have been reported as contributory factors in deaths of mentally handicapped children from conditions requiring emergency surgery. ${ }^{5}$ People with learning difficulties probably experience problems in appreciating or communicating 